\title{
Self-Protection: The Advantage of Radical Oligomeric Mixtures in Organic Synthesis
}

\author{
Hui Yu and Chaozhong $\mathrm{Li}^{*}$
}

Shanghai Institute of Organic Chemistry, Chinese Academy of Sciences, 354 Fenglin Road, Shanghai 200032, P. R. China

clig@mail.sioc.ac.cn

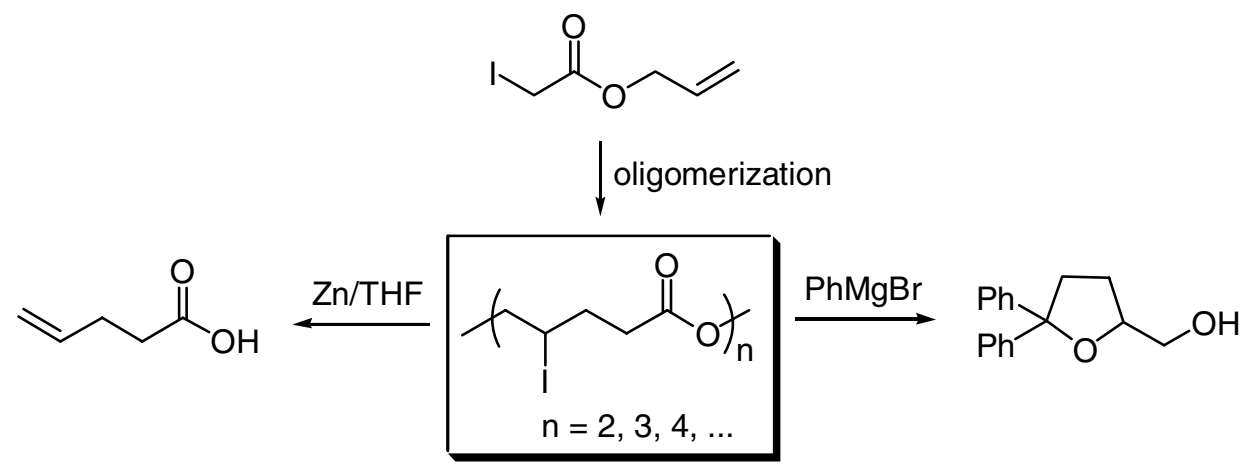

Supporting Information

List of Contents:

1. Characterization of substrate $\mathbf{1 0}$ and references for substrates $\mathbf{1 , 7 - 1 0 , 1 5 - 1 8}$ and products 6a-e.

2. Preparations and characterizations of compounds 25a-c.

3. Characterizations of compounds $\mathbf{1 4 b - 1 4 g}$ and ${ }^{1} \mathrm{H}$ NMR spectra of compounds $\mathbf{1 4 d}$ and $\mathbf{1 4 f}$. 
NMR spectra were recorded in $\mathrm{CDCl}_{3}\left({ }^{1} \mathrm{H}\right.$ at $300 \mathrm{MHz}$ and ${ }^{13} \mathrm{C}$ at $\left.75.47 \mathrm{MHz}\right)$ using TMS as the internal standard. The melting points were uncorrected. All products were isolated by column chromatography on silica gel with hexane - ethyl acetate in an appropriate ratio as the eluent. Methylene chloride was dried over $\mathrm{CaH}_{2}$ and freshly distilled prior to use. THF was refluxed with Na and distilled prior to use. Triethylborane (1 M solution in hexane) was commercially available and used without further purification.

Substrates $\mathbf{1 , 7 - 1 0 , 1 5 - 1 8}$ were prepared according to the conventional methods. ${ }^{1}$

Compounds $1,{ }^{2} 7,{ }^{3} \mathbf{8},{ }^{4} \mathbf{9},{ }^{5} \mathbf{1 5},{ }^{1} \mathbf{1 6},{ }^{6} \mathbf{1 7},{ }^{6} \mathbf{1 8}^{3}$ had the same spectra as in the literature.

1-Hepten-3-yl iodoacetate (10). Colorless liquid. B.p. $78-80^{\circ} \mathrm{C} / 1 \mathrm{mmHg} .{ }^{1} \mathrm{H}$ NMR $(300 \mathrm{MHz}$, $\left.\mathrm{CDCl}_{3}\right) \delta 0.80-0.85(3 \mathrm{H}, \mathrm{t}, J=6.9 \mathrm{~Hz}), 1.19-1.30(4 \mathrm{H}, \mathrm{m}), 1.50-1.63(2 \mathrm{H}, \mathrm{m}), 3.61(2 \mathrm{H}, \mathrm{s}), 5.10-5.25$ (3H, m), 5.65-5.76 (1H, m). EIMS: m/z (rel intensity) $282\left(\mathrm{M}^{+}, 1\right), 169$ (100), 155 (57), 95 (63), 69 (35), 55 (90), 42 (35), 41 (56). Anal. calcd for $\mathrm{C}_{9} \mathrm{H}_{15} \mathrm{IO}_{2}$ : C, 38.32; H, 5.36. Found: C, 38.32; H, 5.39. The spectra of compounds $10 \mathbf{a},{ }^{7} \mathbf{1 0 b},{ }^{8} \mathbf{1 0 c},{ }^{9} \mathbf{1 0 d}{ }^{10}$ and $10 \mathrm{e}^{11}$ synthesized were identical with those reported in the literature.

2,2-Diphenyl-5-(2-hydroxyethyl)tetrahydrofuran (14b). White solid. M.p. $85-86^{\circ} \mathrm{C} .{ }^{1} \mathrm{H}$ NMR $\left(300 \mathrm{MHz}, \mathrm{CDCl}_{3}\right) \delta$ 1.71-2.11 (4H, m), 2.51-2.60 (1H, m), 2.64-2.73 (1H, m), $2.92(1 \mathrm{H}, \mathrm{br})$, 3.86-3.91 (2H, m), 4.28-4.35 (1H, m), 7.17-7.46 (10H, m). ${ }^{13} \mathrm{C} \mathrm{NMR}\left(\mathrm{CDCl}_{3}\right) \delta 31.7,38.1,38.5,61.8$, 79.1, 88.8, 125.7, 125.8, 126.7, 128.1, 128.2, 145.8, 146.4. EIMS: $m / z$ (rel intensity) $268\left(\mathbf{M}^{+}, 10\right)$, 250 (3), 191 (91), 115 (14), 105 (100), 77 (34). Anal. calcd for $\mathrm{C}_{18} \mathrm{H}_{20} \mathrm{O}_{2}$ : C, 80.56; H, 7.51. Found: C, $80.21 ; \mathrm{H}, 7.60$.

2,2-Di(2-propenyl)-5-(2-hydroxyethyl)tetrahydrofuran (14c). Colorless oil. ${ }^{1} \mathrm{H}$ NMR (300 MHz, $\left.\mathrm{CDCl}_{3}\right) \delta 1.55-1.67(1 \mathrm{H}, \mathrm{m}), 1.73-1.84(4 \mathrm{H}, \mathrm{m}), 1.96-2.05(1 \mathrm{H}, \mathrm{m}), 2.21-2.29(4 \mathrm{H}, \mathrm{m}), 3.07(1 \mathrm{H}, \mathrm{t}, J=$ $4.5 \mathrm{~Hz}), 3.77(2 \mathrm{H}, \mathrm{dd}, J=9.6,4.5 \mathrm{~Hz}), 4.07-4.15(1 \mathrm{H}, \mathrm{m}), 5.04-5.09(4 \mathrm{H}, \mathrm{m}), 5.74-5.89(2 \mathrm{H}, \mathrm{m}) .{ }^{13} \mathrm{C}$ 
$\operatorname{NMR}\left(\mathrm{CDCl}_{3}\right) \delta 32.1,33.5,37.4,43.6,44.3,61.6,79.4,85.0,117.7,117.8,134.1,134.2 . \mathrm{EIMS}: \mathrm{m} / z$ (rel intensity) 155 (91), 125 (15), 95 (16), 69 (96), 55 (41). Anal. calcd for $\mathrm{C}_{12} \mathrm{H}_{20} \mathrm{O}_{2}: \mathrm{C}, 73.43 ; \mathrm{H}$, 10.27. Found: C, 73.11; H, 10.24.

2,2-Dimethyl-5-(2-hydroxyethyl)tetrahydrofuran (14d). Colorless oil. ${ }^{1} \mathrm{H}$ NMR $(300 \mathrm{MHz}$, $\left.\mathrm{CDCl}_{3}\right) \delta 1.23(3 \mathrm{H}, \mathrm{s}), 1.26(3 \mathrm{H}, \mathrm{s}), 1.68-1.80(5 \mathrm{H}, \mathrm{m}), 2.04-2.09(1 \mathrm{H}, \mathrm{m}), 3.21(1 \mathrm{H}, \mathrm{br}), 3.77(2 \mathrm{H}, \mathrm{t}, J$ $=5.1 \mathrm{~Hz}), 4.14-4.18(1 \mathrm{H}, \mathrm{m}) .{ }^{13} \mathrm{C} \mathrm{NMR}\left(\mathrm{CDCl}_{3}\right) \delta 28.0,29.2,32.0,37.9,38.0,61.5,78.7,81.3 . \mathrm{EIMS}$ : $m / z$ (rel intensity) $144\left(\mathrm{M}^{+}, 2\right), 129$ (25), 111 (7), 99 (55), 81 (52), 43 (100). HRMS calcd for $\mathrm{C}_{7} \mathrm{H}_{13} \mathrm{O}_{3}$ $\left(\mathrm{M}^{+}-\mathrm{CH}_{3}\right) ; 129.0914$. Found: 129.0927.

2,2-Diphenyl-5-(3-hydroxypropyl)tetrahydrofuran (14e). White solid. M.p 67-68 ${ }^{\circ}$. ${ }^{1} \mathrm{H}$ NMR $\left(300 \mathrm{MHz}, \mathrm{CDCl}_{3}\right) \delta$ 1.64-1.78 (5H, m), 2.01-2.07 (1H, m), $2.38(1 \mathrm{H}, \mathrm{br}), 2.53-2.69(2 \mathrm{H}, \mathrm{m}), 3.73$ (2H, br), 4.13-4.17 (1H, m), 71.6-7.41 (10H, m). ${ }^{13} \mathrm{C} \mathrm{NMR}\left(\mathrm{CDCl}_{3}\right) \delta 30.0,31.6,33.2,38.7,63.0,79.2$, 88.4, 125.7, 125.8, 126.6, 126.7, 128.0, 128.2, 146.4, 146.9. EIMS: $m / z$ (rel intensity) $282\left(\mathrm{M}^{+}, 1\right)$, 264 (14), 223 (10), 205 (65), 183 (31), 165 (18), 105 (100), 77 (27). Anal. calcd for $\mathrm{C}_{19} \mathrm{H}_{22} \mathrm{O}_{2}: \mathrm{C}, 80.82$; H, 7.85. Found: C, 80.59; H, 8.02.

2,2-Diphenyl-5-(2,2-dimethyl-3-hydroxypropyl)tetrahydrofuran (14f). White solid. M.p. 77-78 ${ }^{\circ}$. ${ }^{1} \mathrm{H}$ NMR (300 MHz, $\left.\mathrm{CDCl}_{3}\right) \delta 1.04(3 \mathrm{H}, \mathrm{s}), 1.07(3 \mathrm{H}, \mathrm{s}), 1.29(1 \mathrm{H}, \mathrm{dd}, J=12.6,9.3 \mathrm{~Hz})$, $1.52-1.64(2 \mathrm{H}, \mathrm{m}), 1.75(1 \mathrm{H}, \mathrm{dd}, J=12.3,6.6 \mathrm{~Hz}), 2.44(2 \mathrm{H}, \mathrm{dt}, J=1.8,7.2 \mathrm{~Hz}), 3.46(2 \mathrm{H}, \mathrm{AB}, J=$ $7.8 \mathrm{~Hz}), 3.84(1 \mathrm{H}, \mathrm{s}), 4.02-4.08(1 \mathrm{H}, \mathrm{m}), 7.16-7.46(10 \mathrm{H}, \mathrm{m}) .{ }^{13} \mathrm{C} \mathrm{NMR}\left(\mathrm{CDCl}_{3}\right) \delta 26.5,26.6,30.5$, $38.9,39.6,46.8,77.6,79.8,89.8,126.0,126.2,126.5,126.6,128.0,147.2,147.7$. EIMS: $\mathrm{m} / z$ (rel intensity) $310\left(\mathrm{M}^{+}, 4\right), 293$ (1), 233 (5), 183 (100), 105 (45), 77 (19). HRMS calcd for $\mathrm{C}_{21} \mathrm{H}_{26} \mathrm{O}_{2}$ : 310.1933. Found: 310.1904.

2,2-Diphenyl-5-(4-hydroxybutyl)tetrahydrofuran (14g). Colorless oil. ${ }^{1} \mathrm{H}$ NMR $(300 \mathrm{MHz}$, $\left.\mathrm{CDCl}_{3}\right) \delta 1.43-1.79(8 \mathrm{H}, \mathrm{m}), 1.97-2.04(1 \mathrm{H}, \mathrm{m}), 2.47-2.54(1 \mathrm{H}, \mathrm{m}), 2.59-2.67(1 \mathrm{H}, \mathrm{m}), 3.61(2 \mathrm{H}, \mathrm{t}, J=$ $6.3 \mathrm{~Hz}), 4.05-4.12(1 \mathrm{H}, \mathrm{m}), 7.13-7.45(10 \mathrm{H}, \mathrm{m}) .{ }^{13} \mathrm{C} \mathrm{NMR}\left(\mathrm{CDCl}_{3}\right) \delta 22.4,31.3,32.6,35.9,38.8,62.7$, 
78.9, 87.8, 125.7, 125.8, 126.4, 126.5, 127.9, 128.0, 146.6, 147.2. EIMS: $m / z$ (rel intensity) $296\left(\mathrm{M}^{+}\right.$, 3), 278 (22), 219 (81), 183 (47), 105 (100), 77 (21). Anal. calcd for $\mathrm{C}_{20} \mathrm{H}_{24} \mathrm{O}_{2}: \mathrm{C}, 81.04 ; \mathrm{H}, 8.16$. Found: C, 80.82; H, 8.11.

\section{Typical procedure for the preparations of 25 .}

Triethylborane $(0.2 \mathrm{~mL}, 0.2 \mathrm{mmol}, 1 \mathrm{M}$ solution in hexane) was added to the mixture of methyl iodoacetate $(0.48 \mathrm{~g}, 2.4 \mathrm{mmol})$ and allyl alcohol $(0.25 \mathrm{~mL}, 3.5 \mathrm{mmol})$ in anhydrous $\mathrm{CH}_{2} \mathrm{Cl}_{2}(10 \mathrm{~mL})$ and the resulting solution was stirred at room temperature for $1 \mathrm{~h}$. After removal of the solvent under reduced pressure, the crude product was purified by column chromatography on silica gel with hexane / ethyl acetate $(4: 1, \quad \mathrm{v}: \mathrm{v})$ as the eluent to give the pure product methyl 5-hydroxy-4-iodopentanoate 25a as a colorless liquid. Yield: $538 \mathrm{mg}$ (87\%). IR (film): v ( $\left.\mathrm{cm}^{-1}\right)$ 3450, 1736. ${ }^{1} \mathrm{H}$ NMR $\left(300 \mathrm{MHz}, \mathrm{CDCl}_{3}\right) \delta$ 2.13-2.19 (2H, m), 2.42-2.64 (2H, m), $2.70(1 \mathrm{H}, \mathrm{br}), 3.70(3 \mathrm{H}, \mathrm{s})$, 3.75-3.83 (2H, m), 4.21-4.30 (1H, m). EIMS: $m / z$ (rel intensity) $241\left(\mathrm{M}^{+}+1-\mathrm{H}_{2} \mathrm{O}, 2\right), 131$ (3), 99 (100), 71 (72), 59 (32), 43 (53). Anal. calcd for $\mathrm{C}_{6} \mathrm{H}_{11} \mathrm{IO}_{3}$ : C, 27.93; H, 4.30. Found: C, 28.12; H, 4.31.

5-Acetoxy-4-iodopentanoic acid (25b). Yield: 90\%. Pale yellow oil. IR (film): $v\left(\mathrm{~cm}^{-1}\right)$ 3100, 1741, 1710. ${ }^{1} \mathrm{H}$ NMR $\left(300 \mathrm{MHz}, \mathrm{CDCl}_{3}\right) \delta$ 2.00-2.24 (5H, m), 2.50-2.75 (2H, m), 4.22-4.30 (2H, m), 4.39-4.44 (1H, m), 8.76 (1H, br). EIMS: m/z (rel intensity) 254 (1), 227 (6), 159 (100), 127 (8), 117 (79), 99 (91), 71 (20). Anal. calcd for $\mathrm{C}_{7} \mathrm{H}_{11} \mathrm{IO}_{4}$ : C, 29.39; H, 3.88. Found: C, 29.52; H, 3.95.

Methyl 5-acetoxy-4-iodopentanoate (25c). Yield: 91\%. Colorless oil. IR (film): $v\left(\mathrm{~cm}^{-1}\right) 1738 .{ }^{1} \mathrm{H}$ NMR (300 MHz, $\left.\mathrm{CDCl}_{3}\right) \delta$ 1.97-2.23 (5H, m), 2.41-2.66 (2H, m), $3.69(3 \mathrm{H}, \mathrm{s}), 4.18-4.28(2 \mathrm{H}, \mathrm{m})$, 4.36-4.46 (1H, m). EIMS: m/z (rel intensity) 227 (6), 204 (6), 173 (15), 131 (11), 108 (28), 91 (94), 71 (29), 43 (100). Anal. calcd for $\mathrm{C}_{8} \mathrm{H}_{13} \mathrm{IO}_{4}$ : C, 32.02; H, 4.37. Found: C, 32.19; H, 4.37.

Table S1. ${ }^{1}$ H NMR Spectrum of Compound 14d. 


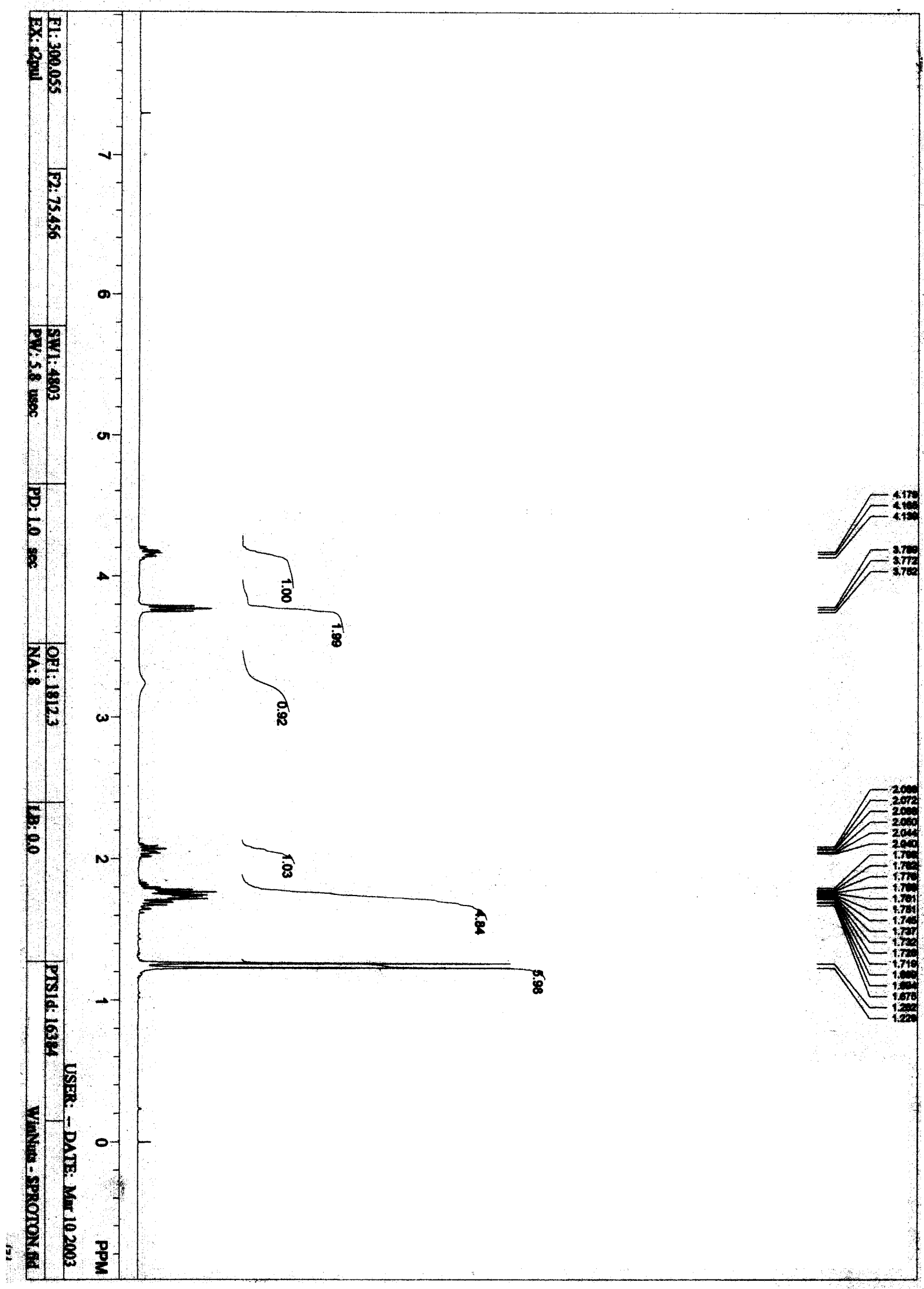

Figure S2. ${ }^{1}$ H NMR Spectrum of Compound $\mathbf{1 4 f}$. 


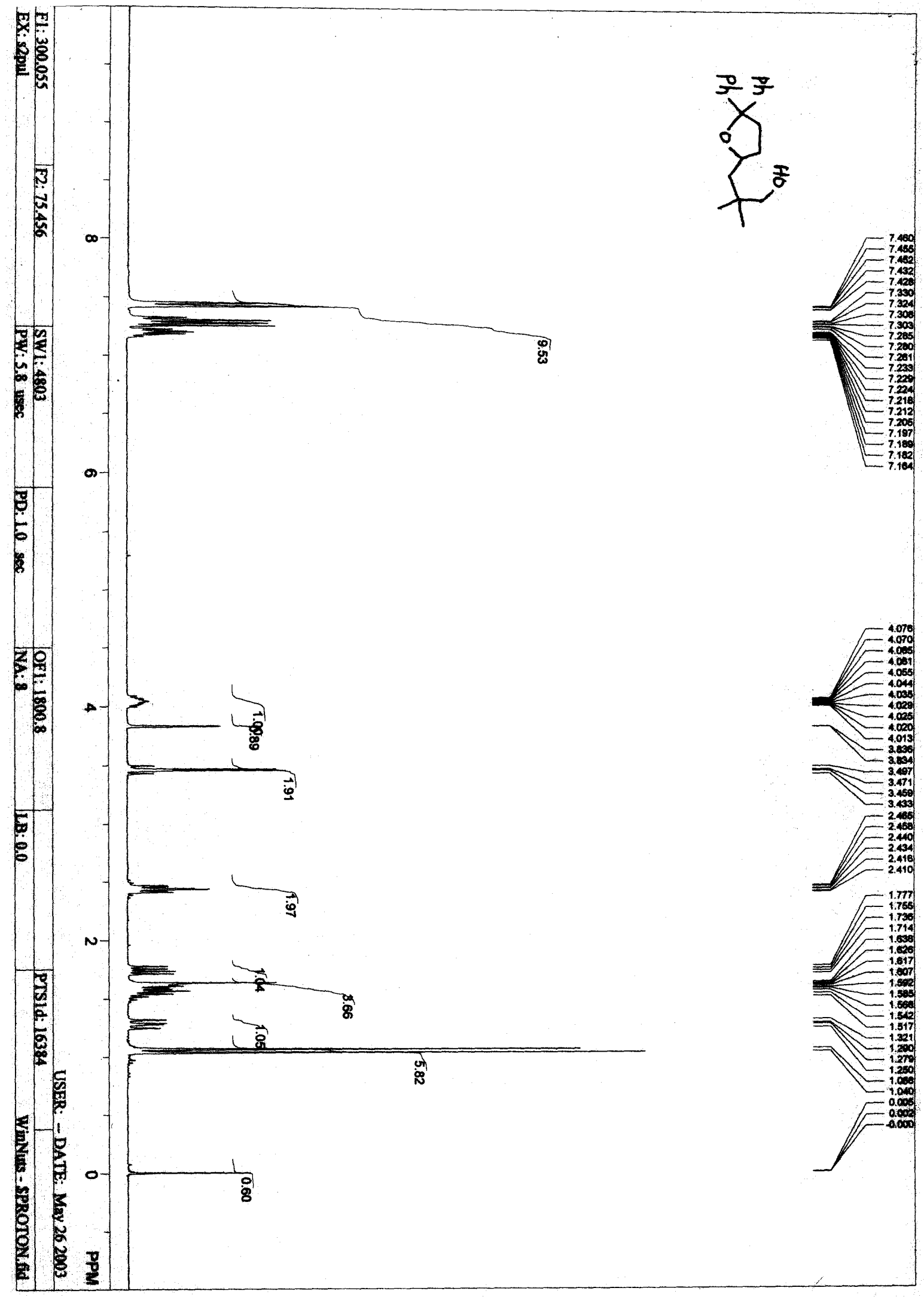




\section{References:}

1. Fang, X.; Xia, H.; Yu, H.; Dong, X.; Chen, M.; Wang, Q.; Tao, F.; Li, C. J. Org. Chem. 2002, 67, 8481 .

2. Curran, D. P.; Tamine, J. J. Org. Chem. 1991, 56, 2746.

3. Yu, H.; Wu, T.; Li, C. J. Am. Chem. Soc. 2002, 124, 10302.

4. Grigg, R.; Sukirthalingam, S.; Sridharan, V. Tetrahedron Lett. 1991, 32, 2547.

5. Yorimitsu, H.; Nakamura, T.; Shimokubo, H.; Oshima, K. J. Org. Chem. 1998, 63, 8604.

6. Wang, J.; Li, C. J. Org. Chem. 2002, 67, 1271.

7. Eisch, J. J.; Merkley, J. H. J. Am. Chem. Soc. 1979, 101, 1148.

8. Artman, G. D.; Waldman, J. H.; Weinreb, S. M. Synthesis 2002, 2057.

9. Baumgarten, H. E. J. Am. Chem. Soc. 1953, 75, 979.

10. Tsuboi, S.; Takeda, A. Bull. Chem. Soc. Jpn. 1975, 48, 1331.

11. (a) Miyano, S.; Hokari, H.; Umeda, Y.; Hashimoto, H. Bull. Chem. Soc. Jpn. 1980, 53, 770. (b) Evans, D. A.; Thomas, E. W.; Cherpeck, R. E. J. Am. Chem. Soc. 1982, 104, 3659. 